\title{
"Doing prescribing": how might clinicians work differently for better, safer care
}

\author{
G Elwyn, A Edwards, N Britten
}

Qual Saf Health Care 2003;12(Suppl 1):i33-i36

Prescribing medicines is a cornerstone of medical practice. There is, however, ample evidence that the prescribing process is far from ideal when viewed from the perspective of patients who wish to understand why they should take medicine, what potential harm they might face, and how they might integrate medicine taking into the pattern of their life, beliefs, and attitudes. Misalignment between clinicians and patients about medicine taking leads to a multitude of problems. Recently, a concept known as concordance has been suggested, where the prescribing interaction is seen as a process where both the patient and professional views and beliefs about medication, and the associated harms and benefits, are shared and negotiated. This interaction depends on a communication process that is becoming known as shared decision making between clinicians and patients. Although there is as yet little evidence that this approach leads to improved clinical outcomes, ethical principles and the core values of medical practice suggest that involving patients in the prescribing process will lead to better, safer care.

See end of article for authors' affiliations

Correspondence to: Professor G Elwyn, Primary Care Group, Swansea Clinical School, University of Wales Swansea, Grove Building, Singleton Park, Swansea SA2 8PP, UK; g.elwyn@ swansea.ac.uk
A amount of prescribed medicine is not taken, or incorrectly used. From the evidence available, we can safely presume that few patients adhere to prescription guidance. ${ }^{1}$ It is also clear that medicine taking is influenced by patients' beliefs and attitudes. ${ }^{2}$ This is particularly true for preventative (and thus largely asymtpomatic conditions), as opposed to curative treatments, and for drugs that have side effects or other drawbacks. As recognition for patient autonomy increases ${ }^{3}$ we are slowly becoming more aware, and respectful, of intentional dissent, where patients decline certain medications on the basis of having been better informed. There has been an important shift in attitude in recent years towards the paradigm of concordance, a paradigm that offers significant opportunities for clinicians to work differently to achieve safer care. Concordance describes a situation where clinicians have identified patients' perspectives, the importance of therapy (if appropriate) has been explained, and an understanding of the consequences of both adherence and non-adherence leads to an agreement about how medication will be used, if at all. This article outlines what is known about large amount of clinical work involves prescribing, yet we know that a significant how best to achieve this agreement, and how to identify the subset of informed patients who do not value specific options and enable them to avoid them. We aim to summarise the evidence about how this task could be improved.

\section{EVIDENCE BASE FOR THIS ARTICLE}

Although there is a substantial body of work that has examined medicine taking, it is a complex area to research. There are few well conducted randomised controlled trials of interventions to help patients follow prescriptions of medications. ${ }^{4}$ This article is based on a number of reviews in this field, ${ }^{145}$ and a recent systematic review of concordance. ${ }^{6}$ Readers should be aware that there have been terminology changes in this area, which have mirrored an increasing rejection of the implicit power gradient in the term to prescribe. The authority laden term compliance gave way to the view that patients adhered (or not) to medication plans. More recently, the term concordance has been used to describe an agreed plan between patient and clinician about the use of medicine, ${ }^{7}$ one of the results of a shared decision making process. ${ }^{8}$

\section{WHAT IS KNOWN ABOUT THE PRESCRIBING PROCESS}

Although the concept of patient centredness has strongly influenced educational curricula, training in the skills of doctor-patient communication has largely concentrated on history taking and diagnosis. Until recently, less attention was being paid to decision making tasks, and it is clear from recent research that patients are rarely involved in these processes. ${ }^{9}{ }^{10}$

Cox et $a l^{6}$ found that doctors initiate discussions about medication but then dominate the interaction. They do not always use the name of the medicine they prescribe or offer descriptions of how new medicines differ in mechanism or purpose from those previously prescribed. They do not usually check patient understanding or explore concerns about medication, and when they encourage patients to ask questions, patients seldom do so. ${ }^{6}$ There is some evidence that clinicians rarely discuss their patients' ability to follow a treatment plan, despite estimating that they do this in about half of their consultations. ${ }^{6}$ In consultations, the benefits of therapy are discussed more often than harms, precautions, or risks, although patients view these latter topics as essential. ${ }^{6}$ Even under formal examination conditions, with added value given to the task of sharing management options with patients, general practitioners fail to demonstrate these attributes on videotape. ${ }^{11}$ In 
summary, clinicians fail to explore the beliefs and hopes of patients about medications, and rarely inform patients about the pros and cons of treatment options. This leaves the territory ripe for misunderstanding, for unaddressed concerns, and for patients to be ambivalent about prescribed medications. ${ }^{12}{ }^{13}$ It is certainly not a sound foundation for informed decisions supported by negotiated, well understood treatment regimes.

\section{THE PATIENT PERSPECTIVE}

The acquisition of a role as one who takes medicine can be a difficult transition. But it seems that many clinicians do not engage with patients' perspectives, ${ }^{12}$ viewing the provision of a diagnosis as substantiation enough that medication is a good thing. There is no doubt that medication is essential to many; taking insulin, hypoglycemic therapy, and thyroxine is vital for millions. Patients rely on taking medicine to curb angina, restrain Parkinson's disease, control asthma, and control other inflammatory diseases in order to maintain reasonable lives. Even so, many patients with chronic illnesses have ambivalence about medication and experiment with titration and drug free intervals. ${ }^{14}$ Given then that patients are circumspect about taking medicine, it is likely that this behaviour will be even more marked when the benefits are less clear, distant, and not of immediate effect. Examples of such situations include the control of blood pressure and the lowering of cholesterol levels. Gaining an understanding of the harms and benefits of using medication for these problems may not go hand in hand with public health interest in reducing population risk and commercial aspirations to obtain returns on investment. If the substantial and modifiable issues of personal risk (body mass, exercise levels, and smoking) were to be addressed first, then the numbers needed to treat for mild hypertension and the primary prevention of cardiac disease are not as persuasive as they might currently appear. ${ }^{15}$

\section{What could be done}

The prescribing interaction is imbued with powerful chemistry; emotional undercurrents that include hope, trust, belief, and confidence. These are the active ingredients (in part at least) of the placebo effect, so we would do well not to abandon the positive hope inducing elements of consultations. But the prescribing process has to change for concordance to be achieved. It is no longer tenable for doctors to prescribe without completing four tasks that seem to be largely neglected: eliciting and exploring patient views, and informing and involving them in the process. In essence, these are skills of sharing decisions (Box 1).

Patients' perspectives on using medicines differ from those of professionals, and as patients may be reticent about voicing their perspectives, these should be elicited early. For example, it is known that patients are inhibited from disclosing prior self treatments, including complementary therapies. Such disclosure is affected by patients' perceptions of the legitimacy of self treatment, which can only be circumvented if clinicians discuss this area directly. ${ }^{17}$ Patients' expectations for prescriptions are influenced by their attitudes to medicines, ${ }^{2}$ but patients' views may not be explored or revealed. ${ }^{12}$ If a patient does not want to take a medicine for a particular problem, this needs to be acknowledged and the reasons discussed. Patients may have a range of concerns about medicines which may or may not correspond to adverse reactions or side effects acknowledged in pharmacological texts.

\section{When is a patient informed}

Does the process of identifying options and sharing information constitute the achievement of informedness? Critics would object, saying that the key outcome is not giving
Box 1 The clinician's competences of shared decision making

- Problem definition-clear specification of the problem that requires a decision from the perspective of both patient and clinician.

- Portray equipoise-that professionals may not have a clear preference about which treatment option is the best, in the context of the patient's priorities.

- Portray options-one or more treatment options and the option of no treatment if relevant.

- Provide information in preferred format-identify patients' preferences if it is to be useful to the decision making process.

- Check understanding-of the range of options and information provided about them.

- Explore ideas, concerns, and expectations about the clinical condition, possible treatment options, and outcomes.

- Checking role preference-that patients accept the process and identify their decision making role preference.

- Decision making-involving the patient to the extent they desire to be involved.

- Deferment if necessary-reviewing treatment needs and preferences after time for further consideration, including with friends or family members, if the patient requires.

- Review arrangements-a specified time period to review the decision.

information, not even information exchange, but patient understanding, that is, do patients comprehend the pros and cons of the therapeutic decision? Researchers have posited that the relevant issues constituting a measure of comprehension (understanding) are the identity and characteristics of relevant health outcomes. Such characteristics include benefits, possible harms, their seriousness, their probabilities, as expressed in absolute and relative terms, and the factors that influence individual susceptibility and the difficulty of avoiding harmful consequences. ${ }^{18}$ Some researchers have sought to identify informed decisions as evidenced by consistency between patients' knowledge, attitudes (to tests or treatments), and their decisions-a so called rational decision making model. However, it is patients, not decisions, whose informedness should be enhanced. This is achieved when decision making (a process not an outcome) is based on an accurate assessment of the information about the relevant decision alternatives and their consequences, an assessment of their likelihood and desirability in accord with the individual's priorities, and importantly a trade off between these factors, ${ }^{19}$ see Box 2 . If this is done, then it enables patients to make reasoned choices about both taking and not taking treatments.

\section{ACHIEVING CONCORDANCE BY SHARING DECISIONS ABOUT THERAPY}

Treatment options may be as simple as to take or not take a medication. When clinicians have addressed patients' concerns, patients understand the potential harms as well as the benefits of the medication, and, as important, the consequences of not taking the medication. The foundation is then laid for involving them in the decision itself. Research 


\section{Box 2 The characteristics of informed decisions}

An informed decision about treatments is one based on:

- an accurate assessment of the information about the relevant decision alternatives and their consequences,

- an assessment of their likelihood and desirability in accord with the individual's priorities,

- a trade off between these factors

into the roles patients wish to take in healthcare decisions is in its infancy. As it develops, it will assess how these roles develop, in both the sense of increasing personal confidence and as patients become expert at managing their condition during their disease career. Significant advances are being made in the study of actual consultations using discourse and conversation analysis. ${ }^{20}$ Such analysis shows that mutuality by patients and doctors is an achievement of both parties and requires the active participation of patients. ${ }^{21}$ There is no doubt, however, that the roles patients do play and those they wish to play are not fixed. They sometimes wish to take on more responsibility than at other times-a reflection of genuine autonomy, but one that is also rooted in a context of an individual's social network or circumstances, and not operating in isolation. ${ }^{22}$ Clinicians need to be aware of changing views about the part medication plays in the undulating patterns of illness and personal perspectives on life. Concordance in other words is a dynamic concept that will require exploration on a repeated basis.

Ascertaining whether patients wish to take part in the decision making process is a critical step. While this may be necessary at various specific decision points (for example, for tests or referrals), taking medication is the ultimate expression of personal decision making and agency. It is something we do to ourselves, so taking medicine regularly is a decision repeated, often indefinitely. An understanding of patients' perspectives is relevant for prescribing even when patients do not want to take part in decision making. Unless clinicians take time to establish patients' underlying conceptions, the chances of supplying prescriptions laden with ambivalences, misunderstandings, and ultimately low motivation for maintaining the regime are rife. Achieving at prescribing interactions where patients are well informed, and satisfied with decisions (whether it is to accept or decline intervention) is, conceptually and pragmatically, a better outcome. Detailed research on this area is required.

\section{RECENT DEVELOPMENTS}

As the recent history of guideline implementation informs us, exhortations have little impact, however prestigious their provenance. There is some evidence that practitioners can improve their communication about medicines and become more skilled at involving patients in decisions and that patient knowledge can be improved. ${ }^{6}$ But these tasks take time. It is not proven that these attributes can be sustained in the pressure of clinical settings. ${ }^{6}$ Multiple interventions, which include the use of patient groups to inform, educate, and self manage, holds promise. ${ }^{23}$ Using administrative staff to monitor medication use $\mathrm{e}^{24}$ and building fail safe repeat prescribing reviews (of under and overuse) are areas that need to be examined in more depth. In the United Kingdom, the Medicines Partnership Task Force is supporting concordance facilitators who can lead local initiatives ${ }^{25}$; an educational resource that helps prescribers to monitor their prescribing has been developed. ${ }^{26}$

\section{Key messages}

- There is extensive variation in the way patients take medicines, which puts patients at risk and leads to significant harm in many cases.

- Medicine taking is strongly influenced by patients' beliefs and attitudes.

- Concordance describes a process where patients and professionals exchange perspectives and beliefs, and achieve agreement about the need (or not) for therapy.

- This process requires that patients are involved in decision making processes.

- Ensuring that patients use medicine effectively may require additional supportive interventions.

- Engaging patients in prescribing decisions so that they understand the risks and benefits of taking and not taking medicine will lead to better, safer care.

\section{POSSIBLE DIRECTIONS}

Health care has to come to terms with the issue of agency, that people behave autonomously. But as Ahearne points out "we fail to recognize that the ways in which we conceive of agency have implications for the understanding of personhood, causality, action and intention". ${ }^{27}$ This is critical in prescribing decisions and behaviours. Doctors who start from the position of recognising, respecting, and enhancing the agency of patients, will, as well as legitimise their own agency as experts, practice differently so as to produce more autonomous patients. To what extent the enhancement of patient agency depends on attitudinal shifts among healthcare professionals, or on the development of widespread patient decision support technologies, is not yet known. Probably both require interventions and innovations to achieve and enhance greater patient engagement in the process of deciding about medicines. This does not need to follow to adherence to treatments-rather, concordant decisions to take or not take treatments must be seen as more desirable. Concordance represents a process by which decisions are made, not necessarily linked to a behavioural outcome. If we achieve such levels of informedness we will have gone a considerable way to involving patients effectively in treatment decisions. Engaging patients in the process in this way is likely to achieve better results for the clinicianpatient relationship and lead to better, safer care for patients.

\section{ACKNOWLEDGEMENT}

This paper is similar to one published in the BMJ: Elwyn G, Edwards A, Britten N. "Doing prescribing": how doctors can be more effective. BMJ 2003;327:864-867.

\section{Authors' affiliations}

G Elwyn, A Edwards, Primary Care Group, Swansea Clinical School, University of Wales Swansea, Grove Building, Singleton Park, Swansea SA2 8PP, UK

N Britten, Institute of Clinical Education, The Peninsula Medical School, St Luke's Campus, Heavitree Road, Exeter EXI 2LU, UK

\section{REFERENCES}

1 Haynes RB, McDonald H, Garg AX, et al. Interventions for helping patients to follow prescriptions for medications (Cochrane Review). The Cochrane Library. Oxford: Update Software, 2003.

2 Britten N Ukoumunne $\mathrm{O}$, Boulton MG. Patients' attitudes to medicines and expectations for prescriptions. Health Expectations 2002;5:256-69.

3 Coulter A. The autonomous patient. London: The Nuffield Trust, 2002.

4 McDonald HP, Garg AX, Haynes RB. Interventions to enhance patient adherence to medication prescriptions: scientific review. JAMA 2002;288:2868-79. 
5 Carter S, Taylor D. A question of choice-compliance in medicine taking. London: Medicine Partnership, Royal Pharmaceutical Centre, 2003.

6 Cox K, Stevenson F, Britten N, et al. A systematic review of communication between patients and health care professionals about medicine-taking and prescribing. London: GKT Concordance Unit, King's College London, 2002.

7 Marinker M. From compliance to concordance: achieving shared goals in medicine taking. BMJ 1997;314:747-8.

8 Elwyn G, Edwards A, Kinnersley P. Shared decision making: the neglected second half of the consultation. Brit J Gen Pract 1999:49:477-82.

9 Elwyn G, Edwards A, Wensing M, et al. Fleeting glimpses of shared decision making: developing the OPTION scale for measuring patient involvement. Qual Saf Health Care 2003; 12:93-99.

10 Stevenson FA, Barry CA, Britten N, et al. Doctor-patient communication about drugs: the evidence for shared decision making. Soc Sci Med 2000;50:829-40.

11 Campion P, Foulkes J, Neighbour R, et al. Patient centredness in the MRCGP video examination: analysis of large cohort. BMJ 2002;325:691-692.

12 Britten N, Stevenson FA, Barry CA, et al. Misunderstandings in prescribing decisions in general practice: qualitative study. BMJ 2000;320:484-8.

13 Barry CA, Bradley CP, Britten N, et al. Patients' unvoiced agendas in general practice consultations: qualitative study. BMJ 2000;320:1246-50.

14 Donovan JL, Blake DR. Patient non-compliance: deviance or reasoned decision-making? Soc Sci Med 1992;34:507-13.

15 Edwards A, Elwyn G, Mulley A. Explaining risks: turning numerical data into meaningful pictures. BMJ 2002;324:827-830.

16 Elwyn G, Edwards P, Kinnersley $P$, et al. Shared decision making and the concept of equipoise: defining the competences of involving patients in healthcare choices. Brit J Gen Pract 2000;50:892-9.
17 Stevenson FA, Britten N, Barry CA, et al. Self-treatment and its discussion in medical consultations: how is medical pluralism managed in practice? Soc Sci Med 2003;57:513-27.

18 Weinstein ND. What does it mean to understand a risk? Evaluating risk comprehension. J Nat Can Inst Mono 1999:25.

19 Bekker H. Genetic testing: facilitating informed choices. In: Encyclopaedia of the human genome: ethical, legal and social issues. Thomas N, ed. New York: Nature Publishing Group, 2003.

20 Drew P, Chatwin J, Collins S. Conversation analysis: a method for research into interactions between patients and health-care professionals. Health Expect $2001 ; 4: 58-70$.

21 Gafaranga J, Britten N. "Fire away": the opening sequence in general practice consultations. Fam Pract 2003;20:242-47.

22 Ashcroft A, Hope T, Parker M. Ethical issues and evidence based patient choice. In: Evidence based patient choice: inevitable or impossible. Elwyn G, ed. Oxford: Oxford University Press, 2001.

23 Bodenheimer T, Lorig K, Holman H, et al. Patient self-management of chronic disease in primary care. JAMA 2002;288:2469-75

24 Simon GE, Von Korff $M$, Rutter $C$, et al. Randomised trial of monitoring, feedback, and management of care by telephone to improve treatment of depression in primary care. BMJ 2000;320:550-554.

25 The Task Force on Medicines Partnership. The task force on medicines partnership. Department of Health, 2003. http://www.medicinespartnership.org.

26 Jenkins L, Britten N, Barber N, et al. Resource pack for reviewing and monitoring prescribing. London: GKT Concordance Unit, King's College, 2002.

27 Ahearn LM. Language and Agency. Annual Review of Anthropology 2001;30:109-37. 